\title{
R\&M 2000 Field Data Requirements for a SPO Operation
}

\author{
Phillip Hermes \\ ASD/ENSSR
}

\begin{abstract}
Traditional and modern perspectives on field data systems are compared with emphasis on the R\&M 2000 and Total Quality Management initiatives which stress operational impacts and customer satisfaction. The Tactical Air Command reporting system is used to illustrate how to develop a "user driven" field data system where TAC's top level metrics (Break Rate, Fix Rate, Ground Abort Rate and Mission Capable Rate) are used as reference points for all levels of data collection, processing and output reports. A matrix of output reports are reviewed which illustrate three levels of reports (Summary, Detail and Raw) for each TAC metric. This matrix approach establishes an "integrated systems" approach to field data systems wherein all reports are interrelated to provide a complete picture to the SPO decision makers.

Selected output displays are reviewed using field data obtained from the TICARRS (F-16 CDS) data system. A Baseline Change Request is being processed by the REMIS SPO to incorporate this approach into the REMIS data system.
\end{abstract}

\section{TRADITIONAL BASE DATA SYSTEM}

The base data system is designed to be used by the base unit for work center management. Base data is generated by different groups at different locations and times. Each group records data for a specific purpose (Scheduling, Debrief, MMICS and Maintenance). This data was written on paper forms, keypunched into card formats and fed into the base computer for processing (Figure 1). The key feature of this data system is that the data recorders were part of the unit which benefitted from the data (responsibilities and resources were in the same organization). This approach prevented excessive data recording requirements for questionable purposes.

After this base data system became operational, the Air Force decided to provide limited off-base data for AFLC materiel management. This was done by mailing MMICS tapes to one analysis community and Maintenance tapes to another comunity. This approach led to data fragmentation where data for a single event (like an aircraft break) was fragmented into Debrief, Maintenance, Status and Utilization data. The operational commands focused on aircraft level data in the Debrief, Status and Utilization data to formulate the Wing Commander's "scorecard". The R\&M, Manpower and Support Cost communities focused on Maintenance data. The ALC Item Managers focused on Status data to identify the equipment supply drivers for aircraft NMCS conditions. The individual communities analyzed "their own" specific data areas without relating "their" data to other data for the same event.

In the meantime, computer capabilities were developed to address the large databases with data links among the data areas. MMICS had limited links and CAMS has considerably more links, which approaches the notion of an "integrated data base".

Later on, the Air Force decided to computerize the base data system by adding the Core Automated Maintenance System (CAMS). The CAMS terminal locations reflect the historical paper reporting locations used for work center management. Any future data system must also support these separate reporting locations. The close association with paper forms also reflects the need to use the paper forms as backup during "offbase" deployment exercises (like Desert Storm).

The point to be made here is that the

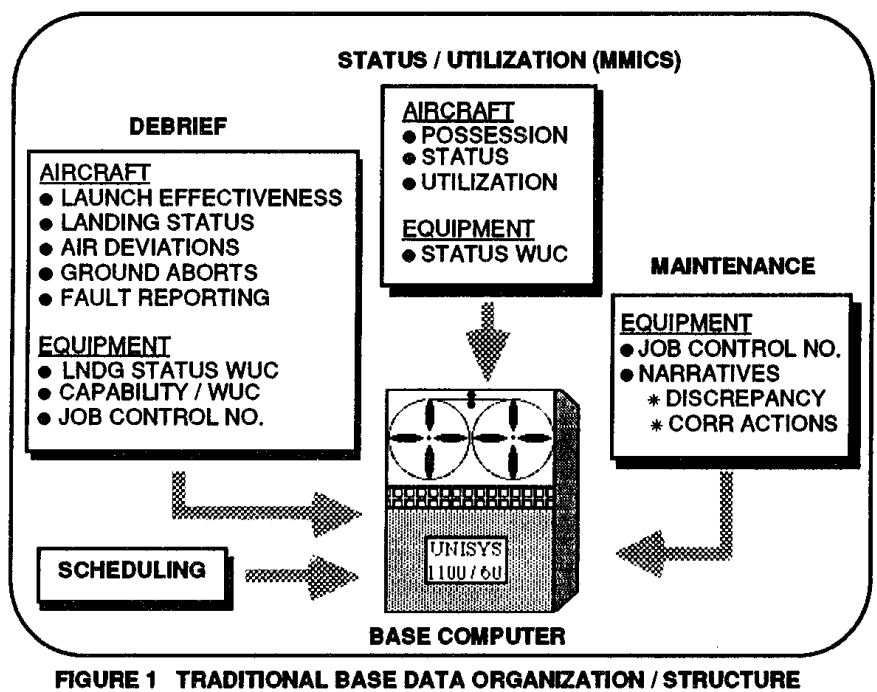


corporate Air Force has allowed field data computer systems to be developed without integrating the base data areas. Field data systems have been developed in the way the data was recorded on paper forms, rather than the way data is needed to make decisions. This condition is largely due to changes in computer technology, where today's computer expectations are unreasonable with respect to the UNIVAC computers used in the 60s.

\section{MODERN FIELD DATA PERSPECTIVES}

Two recent Air Force initiatives (Figure 2) are directly related to the Air Force field data.

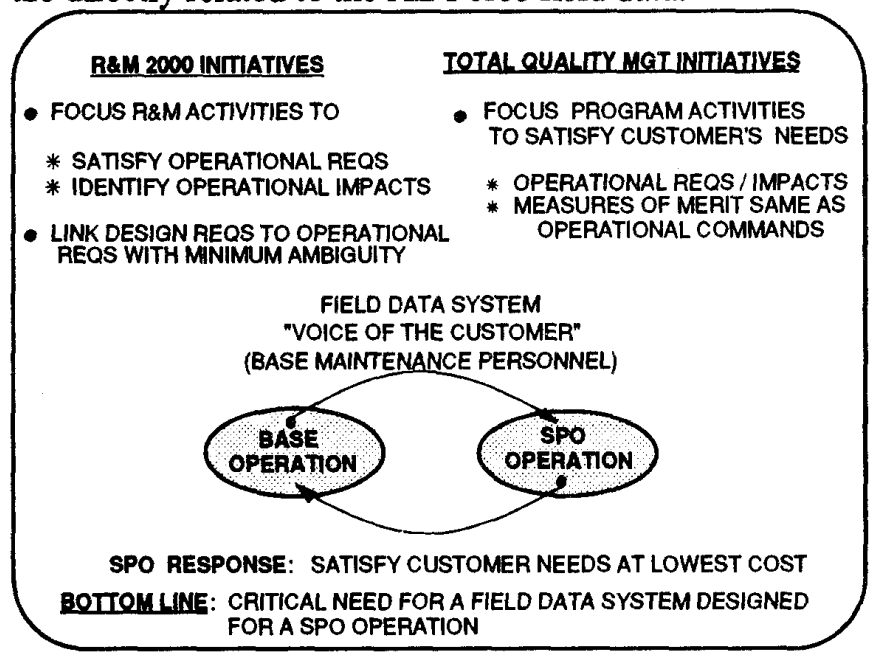

FIQURE 2 MODERN FIELD DATA PERSPECTIVES

The R\&M 2000 initiatives (1986+) were clearly aimed at changing the data processing emphasis from MTBM metrics to operational metrics. The idea was to include operational impacts in the Air Force's decision making data (for example, to prioritize modification funds or fixing field problems). Then, the Total Quality Management initiatives gained momentum $(1990+)$ which also resulted in several AFSC Commander's Policy Letters related to field data which emphasized "Customer Satisfaction".

While it may look strange to identify the maintenance person as the "customer", the actual fact is that the maintenance person is the "customer" from the perspective of $R \& M$, operational readiness, availablility, mission capable rates, sortie generation rates, sustainability, etc. The best way that the corporate Air Force can address these issues by solving the maintenance person's problems. The bottom line, though, is that the traditional Air Force data systems have not been designed for a
SPO operation, so there is no effective communication link between the SPOs and the base maintenance persons they serve.

The rest of this paper is organized to illustrate the characteristics of a "customer driven" field data system, using the Tactical Air Command as an example.

\section{TOP DOWN APPROACH}

The top down approach to field data systems (Figure 3) is the same as the Quality Functional Deployment approach or a Systems Engineering approach which addresses "customer satisfaction". The first step is to scope down the approach to what is most important to TAC. The focus on this approach is the Operational side of TAC (the LG community) and on the most important TAC metrics.

This approach is important for several reasons. It relates all data to critical impacts which enables the SPOs to prioritize corrective actions and funding on the basis of TAC's priorities. It separates the "critical few" issues from the "trivial many" issues (which is important to the corporate SPO organization with limited resources). It focuses on critical operational data which has been "scrubbed" for accuracy at the bases (via briefings, etc.), rather than relying solely on maintenance data, which has not been "scrubbed". Finally, the approach enables all organizations (AFSC, AFLC, TAC and contractors) to focus their efforts in the same direction toward a common goal.

The big challenge is to link the aircraft level data to equipment data (which involves linking Debrief, Status and Maintenance data fields). This challenge is caused by the traditional

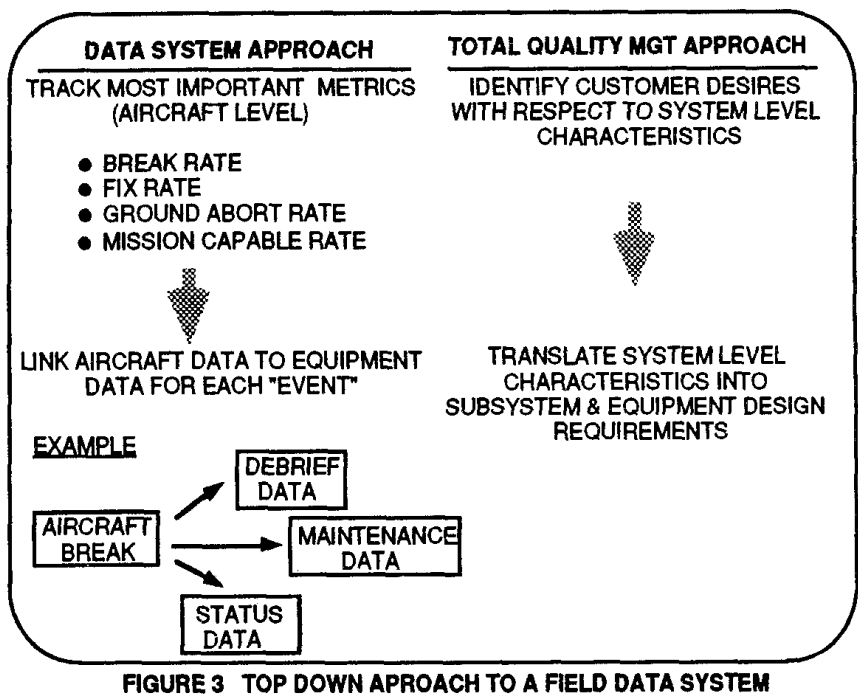


data analysis approach of isolating data into specific areas.

\section{"INTEGRATED SYSTEMS" APPROACH}

An "integrated systems" approach to a field data system is needed to solve field problems. The partitioning shown in Figure 4 is intimately related to a SPO operation, since it relates the aircraft (system) level data to the equipment "drivers" and then provides detailed data on a specific equipment "driver".

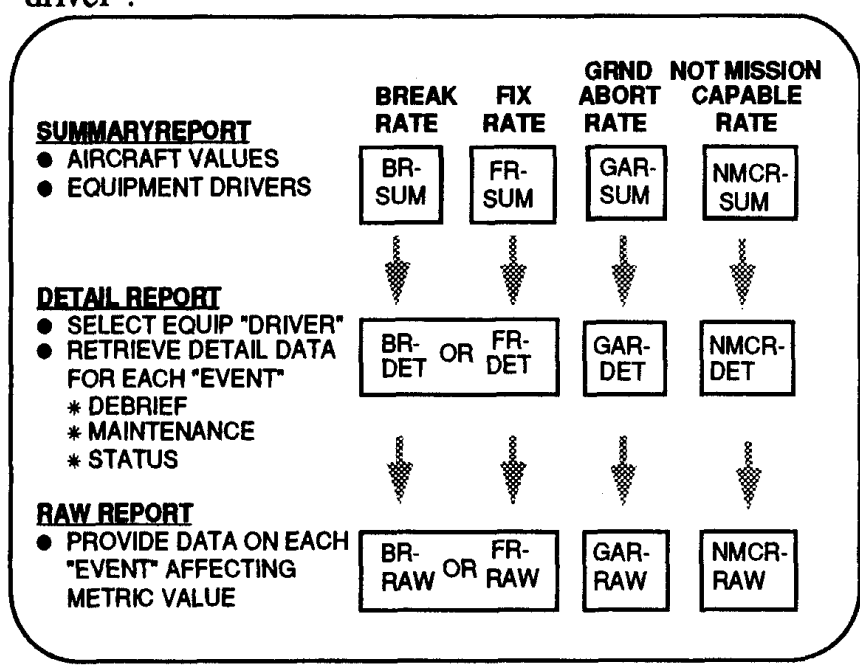

FGURE 4 "WTEGRATED SYSTEMS" APPROACH

The Summary reports are management level reports which indicate the aircraft level value and the equipment "drivers" for each TAC metric. The Mission Capable metric was changed to Not Mission Capable to address FMC problem sources. The Detail reports are for the equipment experts (engineering or logistics...Air Force or contractor) who are familiar with the development or operational history of the equipment. The Detail reports allow the equipment experts to relate their knowledge and experience on a specific equipment to the detailed information in the reports (thus taking advantage of "corporate memory"). The Raw reports provide the raw data which supports the metric calculations. For example the BR-RAW program would list data on each sortie for each Aircraft Maintenance Unit for a visual confirmation of aircraft breaks and sorties. These reports provide the data users with traceability back to the data sources when disagreements arise on the metric values. It also promotes data integrity by highlighting questionable data for corrective actions.

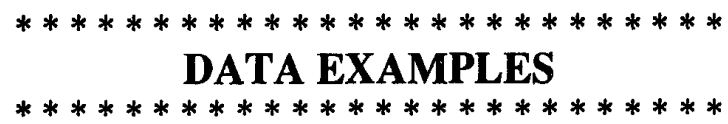

The remainder of this paper provides "real life" examples of field data reports which support the approach reviewed earlier. These reports were developed by programming the TICARRS Query system (with the help of several programming experts in the TICARRS community) to arrive at "custom" reports. Data reports were limited to one base to simplify the programming. Due to space limitations, the report descriptions here are mere skeletons of the actual reports.

Before getting into Break Rate data, I thought it would be a good idea to provide some information on what constitutes an aircraft Break and what it means to TAC. An aircraft Break means that the aircraft cannot be reflown without some repair to restore a function that is mission essential. A Debrief record for this condition will have a Landing Status Code $=3$ ( 2 is minor corrective maintenance and 1 is no maintenance). The aircraft is out of commission until it is Fixed. Break Rate is the number of aircraft Breaks / number of Sorties. The data system problem here is that the Break data is in the Debrief area and the Sortie data is in the Utilization area (and there is no data field linking these two data areas).

TAC's related metric, Fix Time, is the \% of broken aircraft Fixed in 8 hours (TAC analysis disregards the few "outliers" with long Fix times). Fix Time is Non Mission Capable hours (in the Status data area) plus the time between landing (in the Debrief area) and grounding (in the Status area). There are no data fields linking the Fix Time data areas or linking Fix data to Break data.

A point to be made here is that TAC's Break Rate is limited to the aircraft level, while the SPO's work problems at the equipment level. To bridge this gap, I developed a new term called Equipment Break Rate whose values "roll up" to the aircraft break rate value. I define an Equipment break as the equipment system with the 3-digit Work Unit Code (WUC) listed on the Debrief form as the cause of the aircraft break. I also developed a SPO approach to Fix Rate to focus on data that the SPO decisionmakers can relate to. 


\section{BR-SUM DATA}

The summary data for Break Rate is shown in Figure 5. This data is is well suited for management reviews. It provides the managers the minimum essential information (rather than flooding them with less important data).

The Breaks were obtained from the Debrief data, while the sorties were obtained from the Utilization data. In this particular case, the Breaks and Sorties agree with the data in the official TAC 7506 Report (which is most accurate since persons

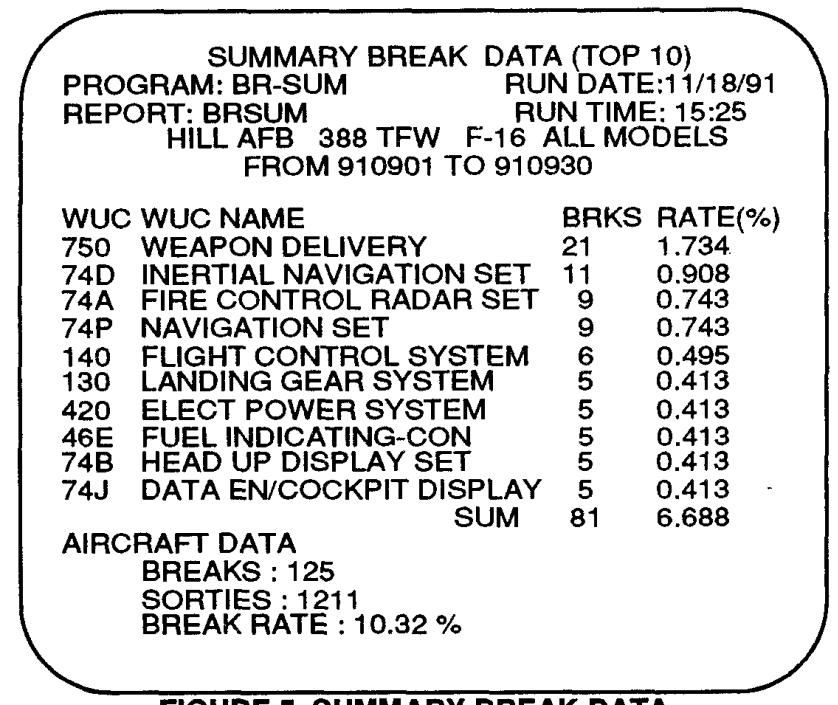

FIGURE 5 SUMMARY BREAK DATA at the bases are dedicated to assure its correctness every day).

An important part of developing a good field data system is to work with the operational commands to develop the right programming algorithms so that the automated data systems are reporting the same metric values as are being reported in the command's internal reporting systems. Given the fragmentation of the present database, this goal is hard to achieve.

From a design / operational perspective, another important characteristic of a good field data system is the ability to select data by aircraft Model Series (A,B, etc.) and Block Numbers to relate $R \& M$ impacts to significant design changes.

\section{BR-DET DATA}

The BR-DET report (Figure 6) is designed to be used to help SPO equipment experts identify what is causing the equipment to be a "driver" for the aircraft metric (in Figure 5). Detailed data is provided for each break for a preselected equipment system (3 digit WUC). This type of data is critical to enable equipment experts to relate their knowledge and experience to the symptoms of the problems. For example, a typical ASD engineer will have about 2 to 4 years experience with the design of the equipment as well as failure modes which occurred during development,

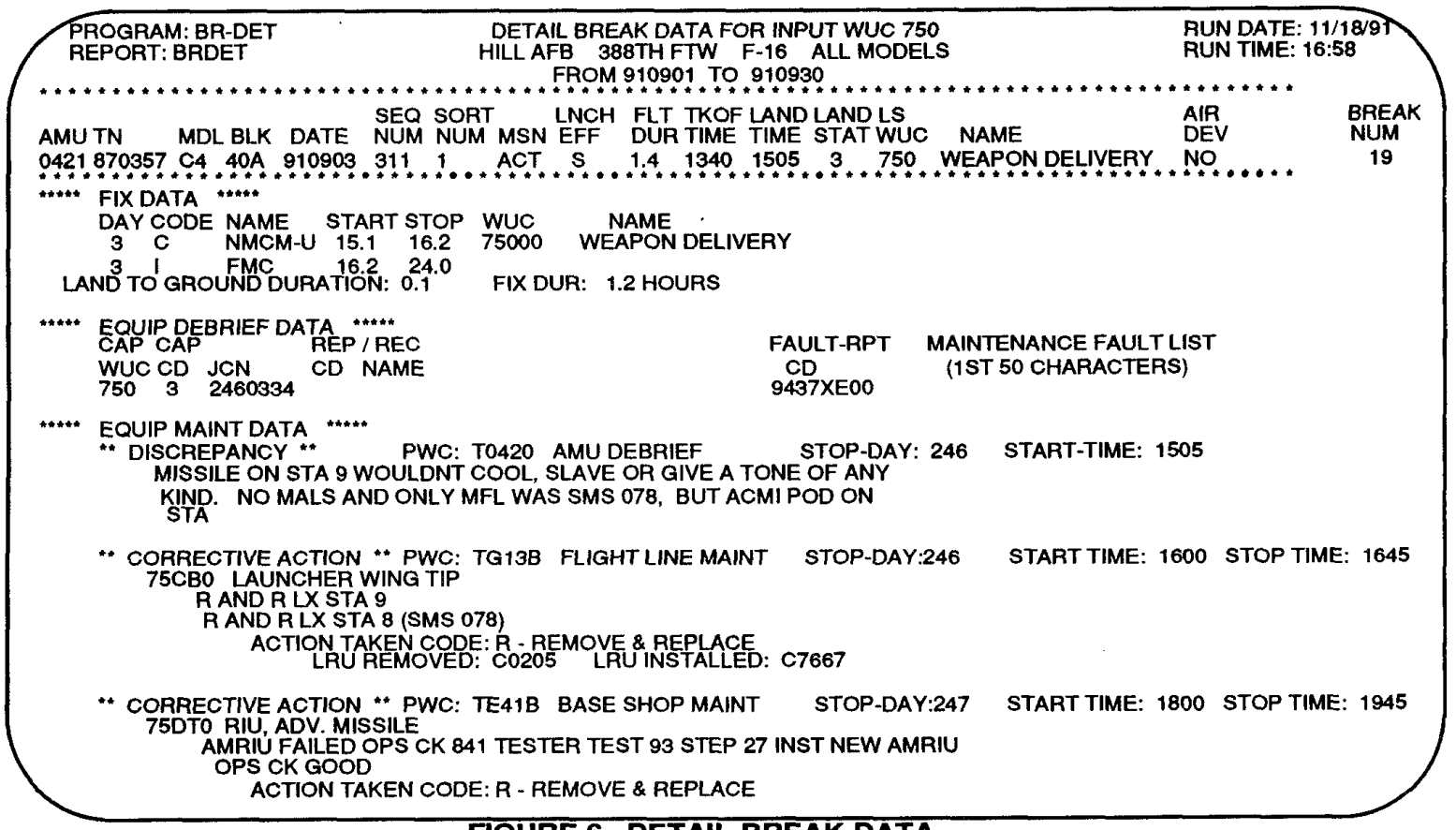

FIGURE 6 DETAIL BREAK DATA 


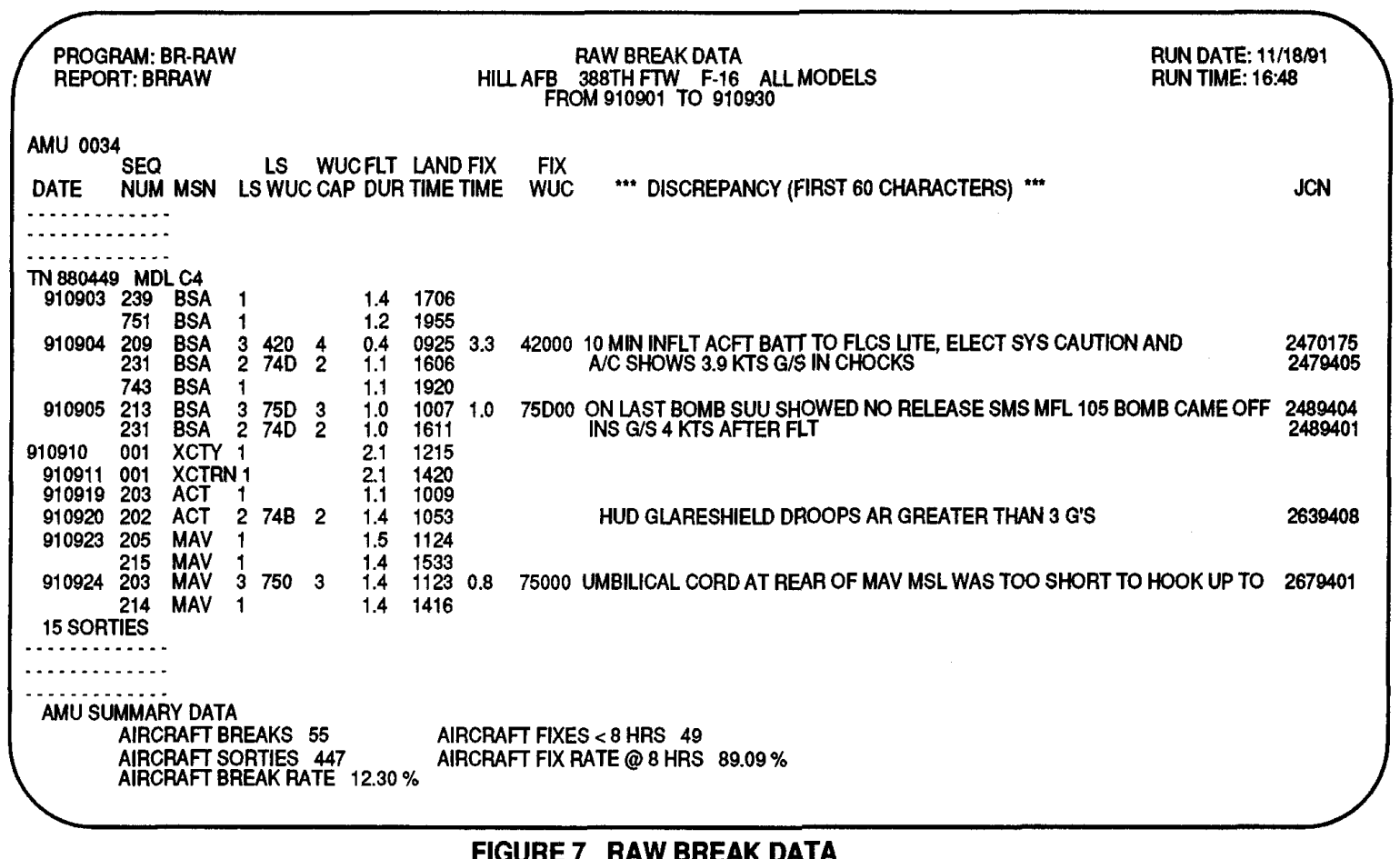

production and flight tests. In order to use this experience to solve field problems, he needs BRDET type data. I chose Break No. 19 for the Weapons Delivery system as an example, since it illustrates both Flight Line and Base Shop data (most breaks for this equipment system were associated with "hung bombs" which were limited to flight-line maintenance). The point to be made here is that all related data needs to be grouped together in one place to enable the data user to comprehend what the data means (i.e., separating Debrief, Status, On-Equip Maint and Off-Equip Maint into separate reports is not a"user friendly" approach).

\section{BR-RAW DATA}

Figure 7 provides an example of a Raw data report. The RAW reports provide the developers and users of a field data system with traceability back to the raw data sources. These reports support the three basic characteristics of a good data system, i.e., Consistancy, Traceability and Agreement With the Official Counting System. Consistency means that the same count occurs on different reports (sorties, maintenance actions, etc.). This issue is also related to programming to eliminate common data recording errors (which can easily be accomplished with a few more lines of code). Traceability means that the summary data (like Break Rate) can be traced back to individual sorties. The data is sorted by AMU, Tail Number, Date and Land time. The AMU sort is critical since the base data is generated by AMU (there can be no reconciliation effort with base personnel without an AMU sort). The number of sorties for each Tail Number is listed to quickly identify where a sortie is missing (when checking against the Utilization records). From a programming perspective, I started with the Utilization records and then tried to find a matching Debrief record. When no match was found, I went to a "library" to match an AMU to the Tail Number and printed a note in the Discrepancy field, "Sortie counted with no debrief. Assume Land Status = 1". The AMU summary data is given at the end of each AMU sortie data to quickly identify if there are any differences between the automated data reporting system and the AMU "in-house" data. The same type of summary is given for the Wing for comparison with the official TAC values.(as recorded in the 7506 base reports to $\mathrm{Hq}$ TAC or the $\mathrm{Hq}$ TAC publications).

\section{FR-SUM DATA}

TAC defines Fix Rate as the \% of aircraft Breaks fixed in 8 hours. While this approach is fine for an operational assessment, a different approach is needed for SPO analysis (to get down 


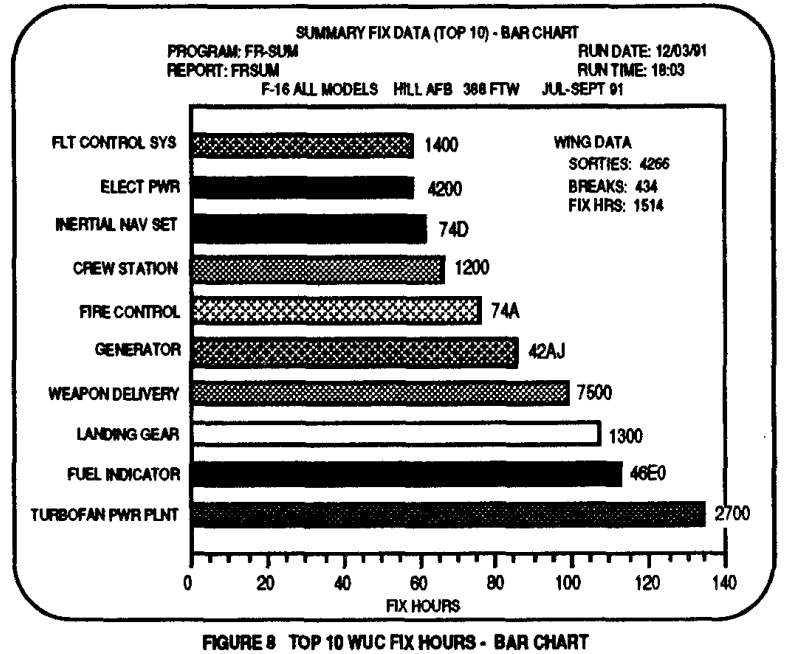

to the equipment "drivers" and to focus the Fix data in a format that SPO decisionmakers can relate to). The SPO approach is illustrated in Figure 8, where the WUCs come from the 5-digit Status WUC associated with aircraft Breaks (I dropped the trailing zero's from this graph). From an analysis perspective, at least 3 months of data is needed to provide an adequate sample size of Fix Times at the 5-digit WUC level of assembly.

The next step in this approach is to determine if the Fix hours are being driven by Break frequency or by long Fix durations. This

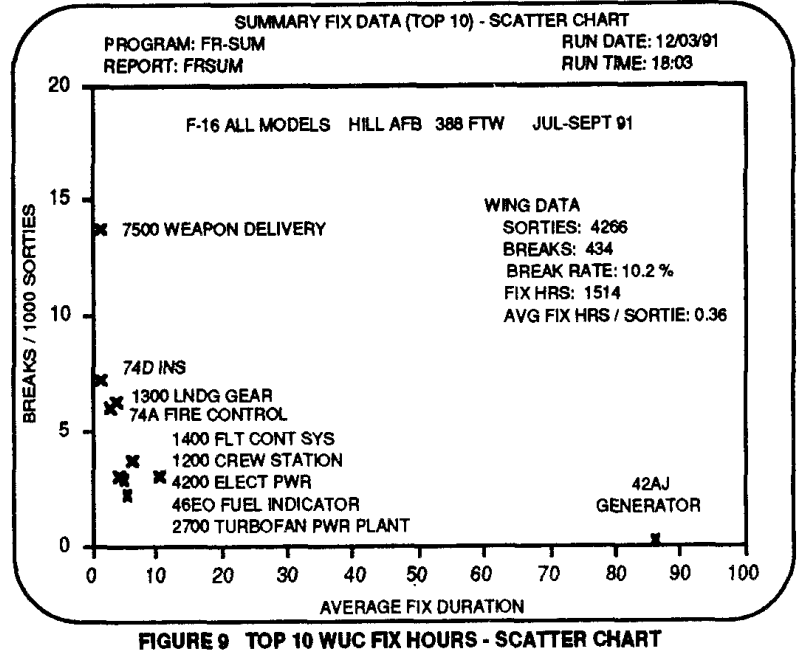

step is illustrated in Figure 9, which indicates that the two most probable equipment candidates to work on are the Weapon Delivery System from a Break Rate perspective, and the Generator, from a Fix duration perspective.

A more detailed look at Summary data is illustrated in Figure 10. This data is sorted by WUC / Tail Number / Date. The WUC's were selected for illustration on the basis of Figure 8 and 9 results (the "integrated systems" approach). The actual report is far more useful for human intelligence than the skeleton data in Figure 10.

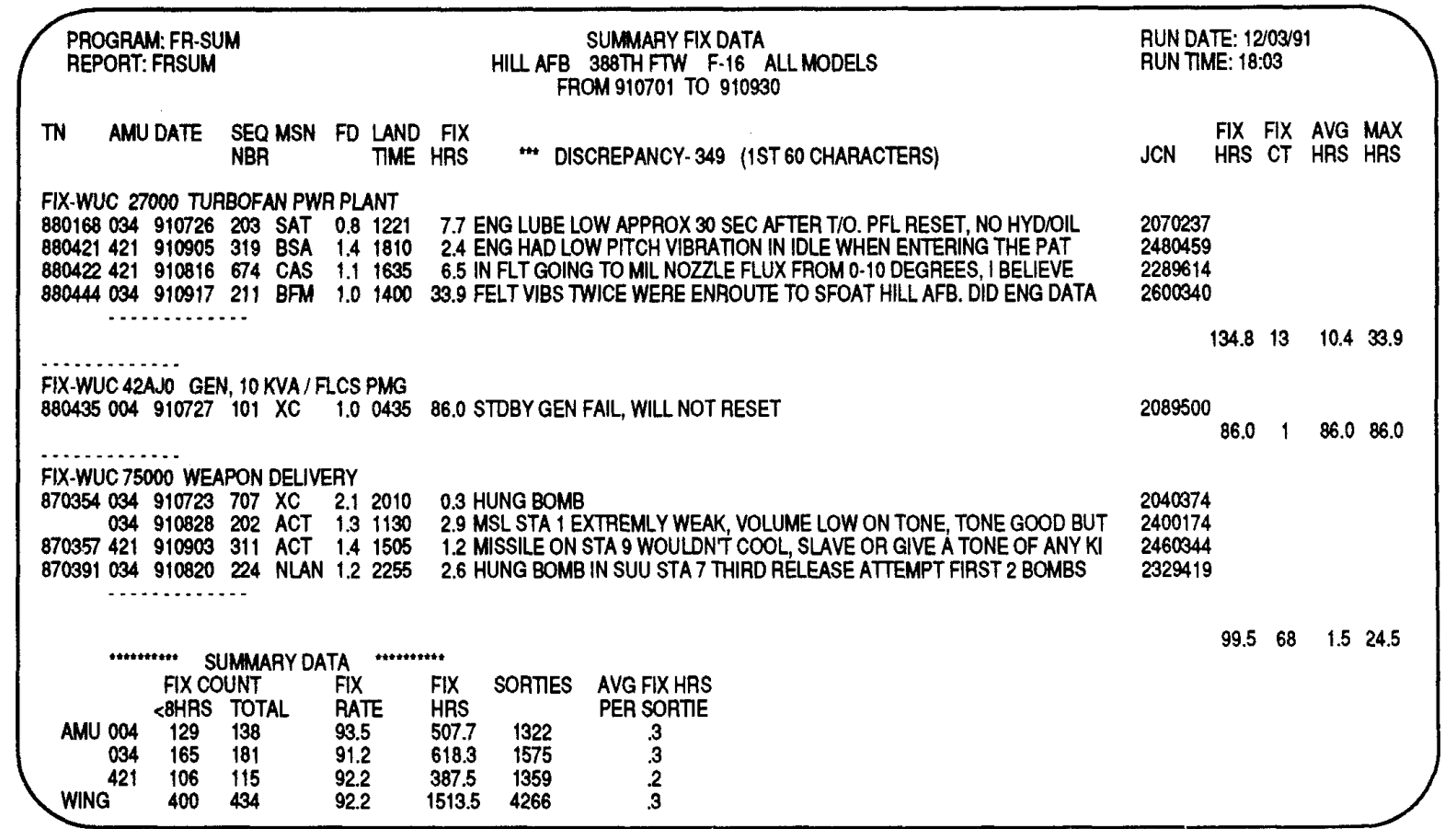

FIGURE 10 FIX SUMMARY DATA 


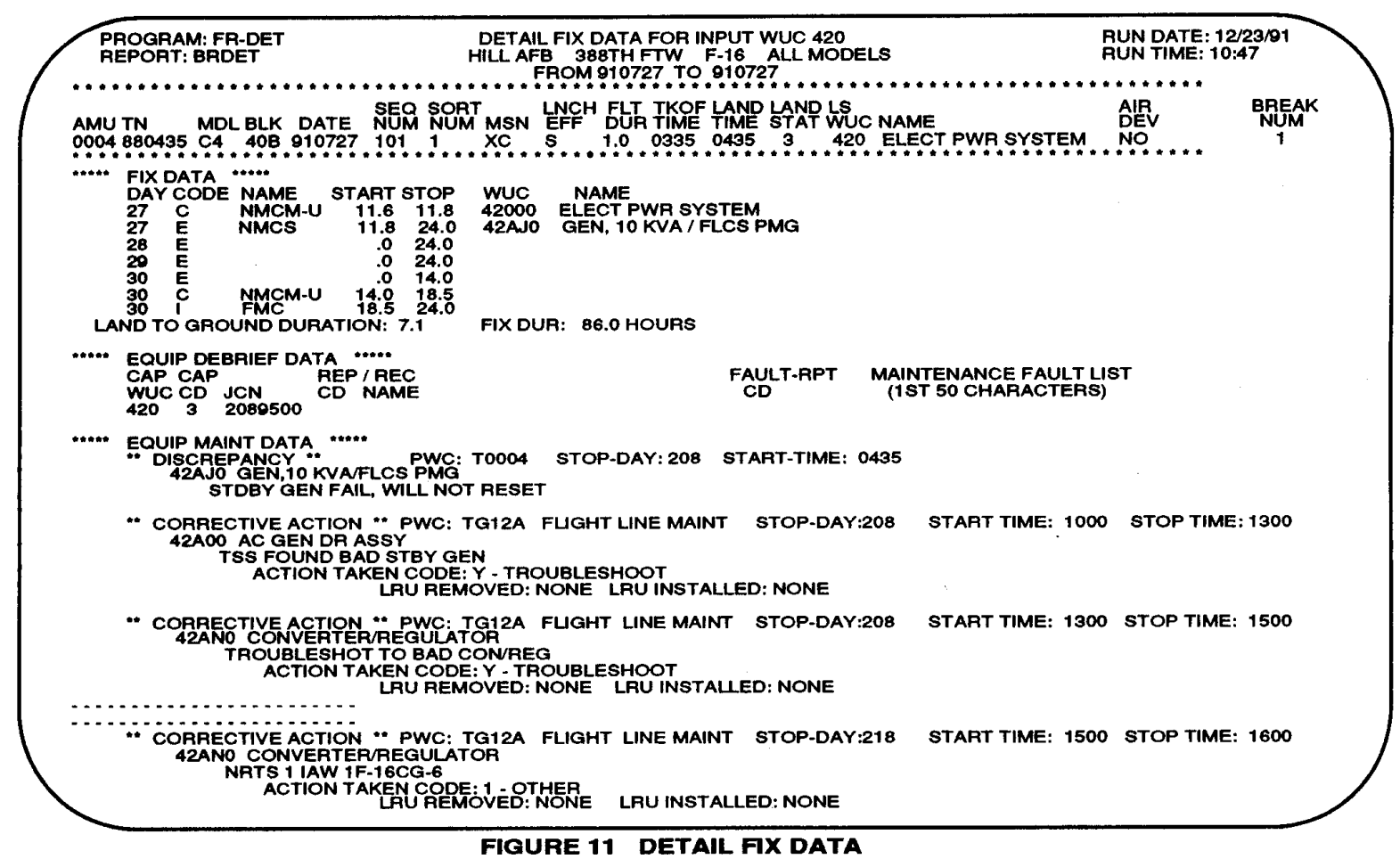

This report characterizes the reported causes of aircraft Breaks and the relative severity of each anomaly on Fix Durations. From a broader perspective, it enables the "user" to apply much more sophisticated logic in analyzing the data. For example, WUC 42000 has 3 "events" which are the same as WUC 42AJ0 (which is reasonable, once you think about it). The point to be made here is that an effective data system provides the correct balance between computer and human processing, but human processing requires specific data content and format to work effectively. The critical part of a computer data system is to define these data needs and provide the minimum essential data in each report.

\section{FR-DET DATA}

The next logical step in this "integrated systems" approach is to try to understand what is driving the "outliers" in Figure 9 (for WUC's 75000 and 42AJ0). The FR-DET data for 75000 is illustrated in Figure 6. The FR-DET data for $42 \mathrm{AJ} 0$ is illustrated in Figure 11. Some of the corrective actions had to be left out to be brief. In this case, the problem seems to be a supply problem associated with the Generator Converter Regulator.

\section{ALL OTHER REPORTS}

Examples for all other reports, listed in Figure 4, are being developed (in a low key, periodic basis which does not interfere with my primary responsibilities to support ASD SPOs in the $R \& M$ areas).

\section{RELATED REMIS BCRs}

The field data approach described herein is included in a REMIS Baseline Change Request (\#289) which was approved by the REMIS CCB on 6 March 91. Its implementation is being delayed to about 1993 (DDN will replace AUTODIN for the REMIS communication system about 1992. AUTODIN has problems with narrative data).

BCR \#289 is being combined with a 1987 TAC. BCR (\#137) which adds the CAMS Debrief Module to REMIS. The intent is to implement the non-narrative portions of BCR \#137 earlier with the narrative portions being implemented with BCR \#289. 